\title{
Sur Jean-Marie Straub-Danièle Huillet, Dalla nube
} alla resistenza

\section{Franco Fortini}

Traducteur : Andrea Cavazzini

\section{OpenEdition}

\section{Journals}

Édition électronique

URL : http://journals.openedition.org/grm/724

DOI : $10.4000 / \mathrm{grm} .724$

ISSN : 1775-3902

\section{Éditeur}

Groupe de Recherches Matérialistes

Référence électronique

Franco Fortini, «Sur Jean-Marie Straub-Danièle Huillet, Dalla nube alla resistenza », Cahiers du GRM [En ligne], 8 | 2015, mis en ligne le 12 décembre 2015, consulté le 30 avril 2019. URL : http:// journals.openedition.org/grm/724; DOI : 10.4000/grm.724

Ce document a été généré automatiquement le 30 avril 2019.

(c) GRM - Association 


\title{
Sur Jean-Marie Straub-Danièle Huillet, Dalla nube alla resistenza
}

\author{
Franco Fortini
}

Traduction : Andrea Cavazzini

\section{NOTE DE L'ÉDITEUR}

Ce texte est paru en 1979 dans le quotidien Il Manifesto avec le titre suivant : « Perché un film aiuta a capire cosa è successo negli ultimi venti anni. E cosa dovra essere » (« Pourquoi ce film nous aide à comprendre les événements de ces vingt dernières années. Et ce qui devra avoir lieu »). Il a été réédité dans F. Fortini, Dissobedienze I. Gli anni dei movimenti. Scritti sul Manifesto 1972-1985, Rome, manifestolibri, 1988, p. 211-215.Toutes les notes de bas de page sont du traducteur.

1 Qu'est-ce que cela signifie d'être convaincu que les œuvres de Straub sont décisives et qu'elles ne nous aident pas moins que les épisodes majeurs de la vie politique à comprendre les événements des vingt dernières années ? Cela veut dire tendre le cou à leur autorité méritée. Mieux: cela veut dire fonder, dans une mesure qui n'est pas moindre, cette autorité-là. Ces œuvres décident, c'est-à-dire partagent, coupent, divisent. Qui ? Elles ne séparent pas seulement ceux qui les rejettent de ceux qui leur donnent leur adhésion - je ne dis pas: leur amour, car elles n'aiment pas et ne se font pas aimer, intolérantes et intolérables. Il ne s'agit pas - il ne s'agit jamais - de goûts subjectifs, mais de manières de voir, et par conséquent de vouloir, le monde. La plus mauvaise réponse à adresser à Straub consiste à dire qu'on aime ses films. La division passe entre deux parties de nous-mêmes : celle qui se confronte à cette violence éthique, et celle qui ne peut la tolérer.

2 Non seulement en tant qu'individu, mais aussi, implicitement, à travers sa façon de traiter ses matières et sa caméra (elle sert chez lui, à faire des prises de ces matières, jamais à en faire des proies $^{1}$ ), Straub émet ses jugements sur l'histoire du cinéma moderne - les jugements d'un "auteur », qui passent sans discontinuité des précisions 
techniques, des menus détails de la fabrication et de la facture de l'œuvre aux perspectives les plus larges des rapports des classes. Par exemple : lorsqu'il nous parle des changements survenus dans la diction des paysans pendant le tournage de la première partie de son dernier film (Dalla nube alla resistenza), tirée des Dialoghi con Leucò de Pavese ; ou lorsqu'il nous renseigne sur le coût du petit troupeau utilisé dans Moses und Aron; ou encore sur le rôle politique très complexe qu'il attribue aux locaux de la radio allemande dans son court-métrage sur Schönberg. On pourrait craindre que cette insistance sur ce qui, dans le film, est "invisible", restant en amont ou à côté du film, relève d'une coquetterie intellectualiste. Il ne fait aucun doute que la volonté de faire en sorte que le spectateur apprenne que tout n'est que travail et reproduction de l'existence, que ce travail est à la fois celui de Bach écrivant sa musique, celui des musiciens qui l'exécutent, celui de l'acteur, intellectuel ou paysan qui récite dans le film, celui du réalisateur et finalement celui du spectateur - il ne fait aucun doute que cette volonté est toujours présente chez Straub, c'est son marxisme, et certaines pages d'Alfred Sohn-Rethel représentent le meilleur commentaire possible de ces films. Cette circulation consciente du travail, c'est-à-dire la transparence du faire dans les choses n'est rien d'autre que le Communisme, en tant que lieu-limite de l'histoire - si ce mot veut toujours dire quelque chose. L'attitude polémique de Straub, et celle de ses films, ont par conséquent un statut prophétique. Pour lui, la malhonnêteté, ou l'erreur, politique, idéologique, philosophique, se fonde ou se réalise dans une malhonnêteté technique, dans l'usage impropre ou frelaté des moyens du cinéma. Son moralisme coïncide avec son formalisme (il en est toujours ainsi). Les conventions qu'il accepte et celles qu'il refuse peuvent paraitre non fondées rationnellement, purement passionnelles, voire des prévarications (ce dont témoigne son peu de disponibilité à faire la part des erreurs et des fautes) : mais elles constituent le code minimal qu'il faut accepter pour le comprendre et travailler avec lui.

3 (Tout cela n'a de sens qu'à condition de le répéter sur le fond des luttes, armées et désarmées, violentes et non-violentes, qui se poursuivent partout dans le monde. Elles se poursuivent aussi contre le silence et le bavardage de tous ceux qui ont trouvé et retrouvent périodiquement, dans les événements locaux et internationaux, des prétextes pour ne pas dire les vérités portant sur l'oppression, ou, pire encore, pour ne pas essayer de savoir laquelle de ces vérités devrait être dite et affirmée la première, et contre quels ennemis. Ils parlent, littéralement, de tout autre chose. Comme si le "cours perverti du monde dont parlait Hegel n'avait depuis toujours fourni assez de justifications tant à la servitude qu'au suicide).

4 Dans le dernier film de Straub il y a quelque chose qui est clair comme un théorème. S'il existe aujourd'hui un "courant» - ou un "reflux », ce mot suave qui garde pour moi le sens qu'il avait à Londres, à l'époque d'Engels : celui de la montée, au rythme des eaux du Tamis, des égouts à ciel ouvert dans les faubourgs prolétariens du East End, - ce film est réellement à contre-courant. D'abord, parce qu'il a rapport à Pavese, un auteur mort il y a trente ans, trop (et mal) lu pendant vingt ans, détesté d'abord par la droite, ensuite par la gauche, considéré aujourd'hui par la morgue des avant-gardes et de l'académisme comme un décadent en retard ou de province. Ce film est à contre-courant parce qu'il parle de la résistance avec un « $\mathrm{r}$ » minuscule, alors même que l'acception historique de ce mot, affublée d'une majuscule, est définitivement souillée, et que personne ne veut réellement savoir ce que " résister » pourrait vouloir dire aujourd'hui. Il est à contre-courant parce qu'il prend position contre les arbres aux sabots et les christs stationnant sur la route entre Eboli-Battipaglia ${ }^{2}$, et parle en revanche de ces paysans sur lesquels tous les dieux 
ont marché, et qui nous ont légué tous les dieux, tout au long de la marche qui relie Néphèle, la Nuée préhistorique, aux Langhes de 1950 dans La lune et les feux.

Dans le dialogue entre Ædipe et Tirésias, les « choses suprêmes » sont dites sur fond du grondement, qui finit par s'imposer, du chariot à bœufs, sans que se perde le sens de l'espace naturel environnant, du chœur secret. Dans la deuxième partie, Nuto et le Bâtard évoquent les événements liés à la Résistance, en marchant entre les témoins muets que sont les arbres et les ravins. Mais Straub dépasse radicalement le discours mythologique de Pavese, incontestablement douteux ; et saisit en revanche le système des pauses et des strophes de son dernier livre, et par là son rythme de complainte et de pitié. L'irréductibilité à laquelle nous invitent les deux personnages - l'un, dans la dernière séquence, par la lecture des pages qui racontent les sacrifices humains dans les feux de 1944 ; l'autre écoutant en contre-champ - n'est pas, comme chez Pavese, le consentement stupéfait face aux cycles du temps et à la fatalité tellurique. Elle est bien plutôt la foi en une lenteur du positif qui n'a de cesse de refaire surface, sous la forme d'une aide et d'une libération réciproques et fraternelles, à travers, et par-delà, les défaites et les horreurs. J'ai écrit «le positif », et j'étais en train d'écrire «l'humain », parce que, dans le langage des années 1940, c'est le mot que Pavese aurait choisi, certes ne prévoyant point, ou plus vraisemblablement n'ayant cure, que quinze ans plus tard, plusieurs auraient ri grassement de ce partage si naif du monde entre les hommes et les autres ${ }^{3}$, et non entre classes en lutte ; mais il pouvait encore moins prévoir, et encore moins en avoir cure, que, une fois passée une deuxième quinzaine d'année, ces mêmes rieurs faciles auraient commencé à déchiffrer et à interroger, sans plus rire, ces textes d'anthropologie et de mythologie que Pavese avait, avec ses moyens, essayé d'introduire chez nous...

6 Je crois que le film de Straub nous fait entendre que la Résistance, qui était il y a plus de trente ans un objet de haine et de dérision pour le curé et pour les clients du bistro - et sur la « vérité » historique de cette séquence il conviendrait de réfléchir - est la même «résistance» détestée aujourd'hui, et d'une manière identique, par les mêmes personnages, peu importe la carte politico-idéologique qu'ils ont dans la poche - je veux dire la résistance que nous opposons, ou devrions opposer, à la démocratie autoritaire, aux technocraties impériales et à la tolérance régressive. Et dans le film tu peux entendre que, tout comme dans notre réalité quotidienne, les armes à feu vont bientôt recommencer à parler.

7 (Tout cela n'a de sens qu'à condition de le reproposer sur fond des formes extrêmes du développement technique et de l'organisation capitaliste du travail, dont parlent les camarades chercheurs déchiffrant notre sort dans les engins très-sophistiqués qui désarment la tradition ouvrière. Les "paysans» de Straub ne sont nullement une énième Arcadie: ils sont la métaphore historique - ou préhistorique - de ce qui se produit sous nos yeux, à Mirafiori, à Arese, après avoir été étudié au MIT ou ailleurs).

8 La tragédie biographique d'un créateur tel que Straub consiste à être forcé d'assister à la formation et au développement de toute une génération d'auteurs intelligents et géniaux qui élaborent et vulgarisent ce qui, dans ses film, a représenté une découverte solitaire. Ainsi, le public commence à s'habituer à des procédés et à un langage auxquels il avait opposé une fin de non recevoir au moment de sa plus grande originalité. Et lorsqu'il sera prêt à déchiffrer Straub il en tirera un sentiment de déjà-vu et il en perdra la signification. Tandis que la "vérité » de Straub ne consiste pas dans les maniérismes techniques que déjà lui ont pu voler ceux qu'un poète appelait «studenti canaglie in versi 
veri $»^{4}$; mais dans le rapport entre ces maniérismes et la «chose » qu'ils disent, entre signifiant et signifié - il en est toujours ainsi, d'ailleurs.

C'est pourquoi les discours compliqués et oraculaires par lesquels certains interprètes de Straub ont voulu exprimer à juste titre leur certitude de l'importance radicale de son œuvre peuvent paraitre et être odieux. Car ces discours finissaient par porter aux nues cette œuvre au sein d'un cercle de connaisseurs exquis, au sein donc de ce que Straub visait à déjouer plus que toute autre chose : le snobisme. Et comme les snobs sont toujours divisés, comme les fourmis, en deux partis, il finissait par être détesté de l'autre espèce du genre des cinématologues. Mais ces films ne s'adressent point à des minorités et à des élites. Ils parlent, tous, d'un dévoilement, donc d'une apocalypse, du monde par-delà sa figure présente. Ils demandent à être appliqués, comme on applique une règle. Mais cela n'est possible que par-delà ces films eux-mêmes. L'anarchisme de Straub n'est pas la fin des hiérarchies qui organisent les valeurs et les êtres; il est la réduction - que les images promettent et anticipent de façon contradictoire - de ce qui est à ce qui devra être, à la nouveauté nécessaire et insoutenable. La réalité que Straub nous présente revêt la même fonction que le pèlerin dans le soir d'Emmaüs: le défunt marchant incognito avec les vivants, qui est reconnu grâce au geste de briser le pain, et qui introduit précisément à travers ce geste la différence absolu dans l'«ici-et-maintenant», en déchirant la vie d'avant et en en ouvrant une autre, côtoyée et exaltée par une absence impossible à combler.

10 (Tout cela n'a de sens qu'à condition de ne pas oublier que les structures de la distribution et de la télévision empêchent le travail de Straub - mais désormais aussi celui de Godard - d'atteindre leurs destinataires, tout en continuant de lui commanditer des films ou de contribuer à ses maigres ressources. C'est une tradition consolidée : éduquer le public à raffoler de certains films - ceux de la grande production, parfois très austères, et que Straub qualifie à juste titre de pornographiques - et à en délaisser d'autres. Comme par hasard, ces derniers sont les seuls qui, sans cris ni gesticulation, montrent comme le monde pourrait être vu).

11 Straub n'a perdu aucun espoir parce qu'il part de ses certitudes, et non de ses espoirs. Il exècre la rhétorique de la "recherche ", du processus ininterrompu et de l'œuvre ouverte - cette forme crapuleuse de " participation ouvrière » à la littérature et à l'art. Il est un cas presque unique d'artiste chez qui convergent la dialectique adornienne du négatif, celle de la prophétie benjaminienne et celle du présent concret que résume le nom de Brecht. Que l'on songe au Bach de son premier film, aux personnages d'Othon, à ceux de Leçons d'histoire, à celui de Fortini/Cani... Quel autre auteur de cinéma, sauf le Dreyer de Gertrud, les a représentés avec autant d'acharnement comme des êtres appartenant à un passé déjà révolu, avec toutes les passions, les idées et les formes de vie qui l'habitaient, tout en les transfigurant en même temps en proposition d'avenir? Je peux me tromper, étant directement impliqué dans son avant-dernier film. Mais il vaut mieux, pour moi et pour qui consent avec moi en me lisant, se tromper sur ces films, sur cette ambition immense, hautaine, parfois repoussante, en dehors des modes et du temps, qu'avoir raison avec le double ordre dominant, qui est à la fois l'apparence de l'ordre et son contraire. 6 mai 1979 


\section{NOTES}

1. [La phrase italienne contient un jeu de mots entre macchina da presa (caméra) et preda (proie)].

2. [Allusion polémique au film d'Ermanno Olmi, L'arbre aux sabots (1978) et sans doute au film de Francesco Rosi (1979) tiré du livre autobiographique de Carlo Levi, Le Christ s'est arrêté à Eboli (1945), qui présentent une image mythologique du monde paysan, idyllique ou paternalistepopuliste].

3. [La phrase italienne est tra uomini e no, allusion au titre du roman d'Elio Vittorini, Uomini e no (1945; titre français, Les hommes et les autres].

4. [Citation d'Eugenio Montale, « Mediterraneo » (1924): « Non ho che queste parole che come donne pubblicate s'offrono a chi le richiede ; non ho che queste frasi stancate che potranno rubarmi anche domani gli studenti canaglie in versi veri»].

\section{RÉSUMÉS}

Ce texte paru en 1979 est un commentaire par Franco Fortini du film des Straub inspiré par les œuvres de Pavese.

\section{INDEX}

Index chronologique : XXe siècle

Mots-clés : Résistance, Brecht, Adorno, Straub, Pavese, Fortini

Index géographique : Europe

Thèmes : esthétique, marxisme 